\title{
The influence of the distribution of cosmic star formation at different metallicities on the properties of merging double compact objects
}

\author{
Martyna Chruslinska, ${ }^{1 \star}$ Gijs Nelemans ${ }^{1,2}$, Krzysztof Belczynski $^{3}$ \\ ${ }^{1}$ Department of Astrophysics/IMAPP, Radboud University, P O Box 9010, NL-6500 GL Nijmegen, The Netherlands \\ ${ }^{2}$ Institute for Astronomy, KU Leuven, Celestijnenlaan 200D, 3001 Leuven, Belgium \\ ${ }^{3}$ Nicolaus Copernicus Astronomical Center, Polish Academy of Sciences, Bartycka 18, 00-716 Warsaw, Poland
}

Last updated 2015 May 22; in original form 2013 September 5

\begin{abstract}
Binaries that merge within the local Universe originate from progenitor systems that formed at different times and in various environments. The efficiency of formation of double compact objects is highly sensitive to metallicity of the star formation. Therefore, to confront the theoretical predictions with observational limits resulting from gravitational waves observations one has to account for the formation and evolution of progenitor stars in the chemically evolving Universe. In particular, this requires knowledge of the distribution of cosmic star formation rate at different metallicities and times, probed by redshift (SFR $(Z, z))$. We investigate the effect of the assumed SFR $(Z, z)$ on the properties of merging double compact objects, in particular on their merger rate densities. Using a set of binary evolution models from Chruslinska et al. (2018) we demonstrate that the reported tension between the merger rates of different types of double compact objects and current observational limits in some cases can be resolved if a SFR $(Z, z)$ closer to that expected based on observations of local star-forming galaxies is used, without the need for changing the assumptions about the evolution of progenitor stars of different masses. This highlights the importance of finding tighter constraints on $\operatorname{SFR}(Z, z)$ and understanding the associated uncertainties.
\end{abstract}

Key words: stars: binaries - stars: black holes - stars: neutron - gravitational waves

\section{INTRODUCTION}

Metallicity is the second most important property, just after mass, determining the stellar evolution. It affects, among others, stellar winds and radii, also impacting the evolution of stars in binaries and the outcome of their evolution (e.g. Maeder 1992; Hurley et al. 2000; Baraffe et al. 2001; Vink et al. 2001; Belczynski et al. 2010a). In particular, the number of close double compact binaries of certain type created per unit of mass formed in stars is known to vary depending on the composition of progenitor stars, the effect being especially significant for double black holes (e.g. Belczynski et al. 2010b; Dominik et al. 2012; Eldridge \& Stanway 2016; Stevenson et al. 2017; Klencki et al. 2018; Giacobbo et al. 2018). Such binaries are the main astrophysical source of gravitational waves that are detected with the currently operating network of ground-based gravitational wave detectors (Abbott et al. 2016b). Using information obtained with detections of gravitational waves from their mergers (e.g. limits on their merger rate density) one can gain insight on the evolution of progenitors of compact binaries. This can be done by confronting theoretically calculated merger rate densities, strongly dependent on the assumptions made in order to describe poorly understood stages of binary evolution (e.g. common

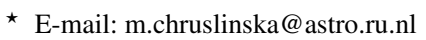

envelope evolution, core-collapse events and related natal kick velocities) with observational limits (see e.g. Chruslinska et al. 2018; Giacobbo et al. 2018; Barrett et al. 2018, for recent results) .

However, since double compact objects (DCO) can form with different parameters (masses, separations, eccentricities), they need different amount of time to merge due to gravitational wave radiation (e.g. Peters 1964). As a consequence, binaries formed at different times, in different environments and hence with different metal content all contribute to the merger rate we measure locally. The number of merging binaries depends on the amount of star formation happening throughout the cosmic time (probed by redshift) but also on the distribution of the star formation rate across different metallicities $(\operatorname{SFR}(\mathrm{Z}, \mathrm{z})$; since the evolution is metallicity dependent). To estimate the merger rate density it is necessary to assume a certain history of star formation and chemical evolution of the Universe, which adds another layer of uncertainty to those calculations.

Alterations in the assumed $\operatorname{SFR}(\mathrm{Z}, \mathrm{z})$ also change the properties of the locally merging population of DCO. Since the dependence of the formation efficiency of merging double compact objects on metallicity is different for different types of binaries, any change in $\operatorname{SFR}(Z, z)$ particularly affects the ratio of their merger rates. Moreover, as the stellar wind mass loss is a function of metallicity, changing $\operatorname{SFR}(Z, z)$ will have a significant effect on the distribu- 
tion of masses of the locally merging double black holes.

Recently, Chruslinska et al. (2018) (hereafter C18) demonstrated that the local double neutron star (NSNS) merger rate densities typically fall significantly below the current lower limit implied by gravitational wave observations (Abbott et al. 2017c). Within a set of 21 models calculated with the StarTrack population synthesis code (Belczynski et al. 2002, 2008) they identify three, requiring quite extreme assumptions about the evolution of progenitor stars that lead to NSNS merger rates consistent with this limit. However, the associated double black hole merger rate densities calculated for those models exceed the upper limit on their merger frequency set by LIGO/Virgo observations (Abbott et al. 2017a). We argue that the assumed distribution of the cosmic star formation at different metallicities and redshifts used in this study significantly over predicts the amount of star formation happening at low metallicities. We use their models as an example to demonstrate the consequences of different assumptions on $\operatorname{SFR}(\mathrm{Z}, \mathrm{z})$ for the properties of merging DCOs. We show that in two out of three cases the reported discrepancy may be resolved if a different $\operatorname{SFR}(Z, z)$ with higher metallicity of the star formation is used.

Throughout the paper we adopt a standard flat cosmology with the following cosmological parameters: $H_{0}=70 \mathrm{~km} \mathrm{~s}^{-1} \mathrm{Mpc}^{-1}$, $\Omega_{M}=0.3, \Omega_{\Lambda}=0.7$ and $\Omega_{k}=0$.

\section{MERGER RATE DENSITY AND METALLICITY}

The rate of DCO mergers is strictly connected to the pace of the cosmic star formation (SFR(z)). The higher the SFR(z), the more DCO mergers. Those mergers occur with a certain delay in time, which is needed to complete the evolution of stars to the point where two compact objects coalesce. In general, this time depends on the binary parameters (and as such is metallicity dependent). The distribution of DCO delay times $t_{d e l}$ is typically strongly peaked at short times $(\sim 100 \mathrm{Myr})$ and falls off as $\sim t_{d e l}^{-\alpha}$, with $\alpha$ being of the order of unity. Knowing this distribution, one can calculate what fraction $\left(\mathrm{f}_{\mathrm{loc}}^{\mathrm{mr}}\right)$ of merging systems formed at redshift $z$ with metallicity $Z$ merges in the local Universe (at redshift $z \leqslant z_{\text {loc }}$ or equivalently within $\delta t_{l o c}=t_{0}-t\left(z_{\text {loc }}\right)$, where $t(z=0)=t_{0}$ is the Hubble time). However, since the efficiency of formation of merging DCOs $\left(\chi_{\mathrm{DCO} ; \mathrm{i}}\right)^{1}$ is a function of metallicity, it is not enough to know the absolute star formation rate $(\operatorname{SFR}(\mathrm{z}))$, but rather its distribution at different metallicities $\operatorname{SFR}(Z, z)$ or a fraction of $\operatorname{SFR}(Z)$ that at each $z$ happens at a certain metallicity $\left(f_{\text {sfr }}(z, Z)\right)$.

Thus, the local merger rate density of DCOs of certain type (double neutron stars - NSNS, double black holes - BHBH, neutron star black hole binaries BHNS/NSBH) can be expressed as:

$$
\begin{array}{r}
\mathrm{R}_{\mathrm{DCO} ; \mathrm{i}}^{\mathrm{loc}}=\frac{1}{\delta \mathrm{t}_{\mathrm{loc}}} \sum_{\mathrm{z}} \sum_{\mathrm{Z}}\left(\chi_{\mathrm{DCO} ; \mathrm{i}}(\mathrm{Z}) \mathrm{f}_{\mathrm{sfr}}(\mathrm{z}, \mathrm{Z}) \frac{\mathrm{SFR}(\mathrm{z})}{\delta \mathrm{V}} \times\right. \\
\left.[t(z+\delta z)-t(z)] f_{l o c}^{m r}(z, Z)\right)
\end{array}
$$

where the sum runs over redshifts $(\mathrm{z})$ and metallicities $(\mathrm{Z})$ at which the progenitor stars form and $\delta V$ is the comoving volume element. Note that $\chi_{\mathrm{DCO} ; \mathrm{i}}$ is model $^{2}$ dependent. Modifications of the

\footnotetext{
${ }^{1} \chi_{\text {DCO;i }}$ is defined as a number of merging double compact objects of certain type created per unit of mass formed in stars

2 in population synthesis studies a model is defined by the choice of a particular set of assumptions (parameters) used to describe the evolution of binaries, e.g. conservativeness of the mass transfer, distribution describing the magnitude of NS and BH natal kicks
}

assumptions made to describe evolution of DCO progenitors result in changes in $\chi_{\mathrm{DCO} ; \mathrm{i}}$ and hence in the estimated merger rates, e.g. if more neutron stars are allowed to form with relatively small natal kick velocities, the formation efficiency of merging double neutron stars generally increases. However, certain modifications can boost or decrease $\chi_{\text {DCO;i }}$ only in specific metallicity range (e.g. assuming fully-conservative mass transfer as discussed in Dominik et al. 2012, C18 (models V12 and J5 respectively) affects mostly the number of merging NSNS formed at high metallicity).

For a given model, $\chi_{\mathrm{DCO}}(\mathrm{Z})$ also depends on the choice of distributions used to describe the initial parameters of binaries (de Mink \& Belczynski 2015), although the change is minor, unless the high mass tail of the initial mass function is allowed to vary with metallicity (see fig. 6 in Klencki et al. 2018). Despite this sensitivity of $\chi_{\mathrm{DCO}}(\mathrm{Z})$ on the model assumptions, certain characteristics seem robust (see Giacobbo et al. 2018 and sec. 4.2 in Klencki et al. 2018). For instance, BHBH form much more efficiently at low metallicities than at high $Z$ and $\chi_{\mathrm{BHBH}}(Z)$ reveals a sharp decrease (a factor of $\gtrsim 10)$ at $Z$ approaching solar values. $\chi_{\mathrm{NSNS}}(Z)$ usually shows much smaller variation with metallicity and increases slightly towards higher $Z^{3}$. Generally, $\chi(Z)$ evolves differently for different types of DCOs, hence any change in $f_{\text {sfr }}(z, Z)$ would affect the ratios of merger rates of binaries of different type.

Different approaches have been taken to determine $\mathrm{f}_{\text {sfr }}(\mathrm{z}, \mathrm{Z})$ used to calculate merger rate densities. One way is to extract this information from cosmological simulations (e.g. Mapelli et al. 2017; Schneider et al. 2017), the other is to use the available observations and/or complement observational results with theoretical inferences (Dominik et al. 2013; Belczynski et al. 2016a; Eldridge et al. 2018, Chruslinska et al. in prep.).

All methods have their shortcomings. Cosmological simulations do not fully reproduce all of the observational relations (e.g. mass - metallicity relation) and are resolution-limited. Observations on the other hand are subject to biases and provide complete information only in very limited ranges of redshifts and luminosities of the objects of interest. In any case, the use of incorrect SFR $(Z, z)$ clearly affects the resulting cosmological merger rates and may lead to erroneous conclusions. However, the importance of the assumed $\operatorname{SFR}(\mathrm{Z}, \mathrm{z})$ for calculated $R_{l o c}$ was not quantified in previous studies. Here we focus on the method introduced by Belczynski et al. (2016a) (hereafter B16; see appendix on method therein), as it was also used by $\mathrm{C} 18$ whose models we use in this study.

B16 use the cosmic SFR density from Madau \& Dickinson (2014) and the mean metallicity of the Universe as found by these authors increased by 0.5 dex to better represent the metallicity at which the star formation occurs. This metallicity was used as a mean of the metallicity distribution $\left(Z_{\text {avg }}\right)$, described as a log-normal with a substantial scatter of $\sigma=0.5$ dex. Despite the applied shift, the $Z$ of star formation in B16 is likely underestimated. Observations suggest that massive galaxies dominate the star formation budget in the Universe and the star forming gas found in those galaxies has relatively high metal content (which is close to, or higher than the solar value $\mathrm{Z}_{\odot}$, even if uncertainty in the absolute metallicity calibration is taken into account, e.g. Kewley \& Ellison 2008).

According to the assumption made by B $16 \sim 70 \%$ of stars at certain redshift form with $\log (\mathrm{Z})$ in the range $\log \left(\mathrm{Z}_{\text {avg }}\right) \pm 0.5$ dex (i.e. range

\footnotetext{
${ }^{3}$ but see models $\mathrm{J} 1 B, \mathrm{~J} 7 B$ and $\mathrm{J} 5 B$ in $\mathrm{C} 18$, where $\chi_{\mathrm{NSNS}}(\mathrm{Z})$ decreases at high $Z$ - this can be seen by comparing the numbers in column 3 from their table 2, However, note that those models significantly underpredict the Galactic merger rates
} 
of $1 \mathrm{dex}$; this scatter around $\log \left(\mathrm{Z}_{\text {avg }}\right)$ is assumed to be constant throughout the cosmic history). Using the stellar mass - metallicity relation for galaxies (MZR) by Tremonti et al. (2004), it can be seen that the range of metallicities corresponding to galaxy stellar masses $\mathrm{M}_{*} \gtrsim 10^{9} \mathrm{M}_{\odot}$ (which are responsible for $\sim 70 \%$ of the local star formation) is around 0.5 dex. ${ }^{4}$ Taking into account the intrinsic scatter in the MZR of $\sim 0.1$ dex this range may be broadened to 0.7 dex. This naive estimate suggests that the scatter around $Z_{\text {avg }}$ may be smaller than what was assumed in B16 (at least in the local Universe, as the amount of scatter in the relation may in principle be redshift dependent). However, the metallicity gradients within galaxies may also contribute to the scatter in $\mathrm{Z}$ at which the stars form at a given redshift. Their contribution is difficult to constrain as the results vary significantly between studies and are likely affected by the adopted metallicity calibration and can be mass dependent (e.g. Sánchez-Menguiano et al. 2016; Belfiore et al. 2017; Poetrodjojo et al. 2018).

In this study we introduce two simple modifications to the assumptions made by B16 to investigate what would be the effect of higher $Z_{\text {avg }}$ and smaller scatter around this metallicity on final results.

\section{METHOD}

We take three models $(\mathrm{NK} 2 A, \mathrm{C} A, \mathrm{C}+\mathrm{P} A)^{5}$ from $\mathrm{C} 18$ as an example. Those models were found to satisfy the current limits on $R_{l o c}$ for NSNS systems implied by GW170817 (Abbott et al. 2017c), at the same time violating the associated upper limit for $R_{l o c}$ of BHBH (Abbott et al. 2017a). Briefly, the differences in these models compared to the reference model from $\mathrm{C} 18$ are

- in model $N K 2$ half of the iron-core collapse supernovae was assumed to lead to small natal kick velocities $\leqslant 50 \mathrm{~km} / \mathrm{s}$

- model $C$ incorporated several modifications found to favour the formation of merging DNS: Bray \& Eldridge (2016) ${ }^{6}$ prescription for the natal kicks that depends on the amount of mass ejected during the supernova and the mass of the remnant (as opposed to the distribution proposed by Hobbs et al. (2005) used in the reference model, that is independent of the characteristics of the star undergoing supernova), reduced angular momentum loss during the mass transfer and wider limits on the helium core mass for the progenitors of stars undergoing electron-capture supernovae;

- model $C+P$ adds to model $C$ the assumption that mass transfer in systems with Hertzsprung gap donors and NS/BH accretors is stable and never than leads to common envelope. In case of other types of accretors, common envelope evolution was allowed.

In all three models the common envelope evolution with Hertzsprung gap donors was allowed (variation $A$, as opposed to variation $B$ from $\mathrm{C} 18$ where those cases were assumed to lead to merger). For more details we refer the reader to the original paper. The formation efficiencies $\chi(\mathrm{Z})$ for those models are shown in fig. 1. Note that the simulations were performed for a discrete set of 32 metallicities (listed in B16) and we assume that the formation

${ }^{4}$ This range is likely conservative, since MZR found by Tremonti et al. (2004) is among the steepest MZRs present in the literature (see Kewley \& Ellison 2008).

${ }_{5} R_{l o c}$ calculated for those models can be found in tables 2 and 3 in $\mathrm{C} 18$

6 Note that after the publication of C18, Bray \& Eldridge (2018) updated the natal kick model given in Bray \& Eldridge (2016). Adopting their updated prescription was found to significantly increase the predicted DNS merger rates Eldridge et al. (2018).

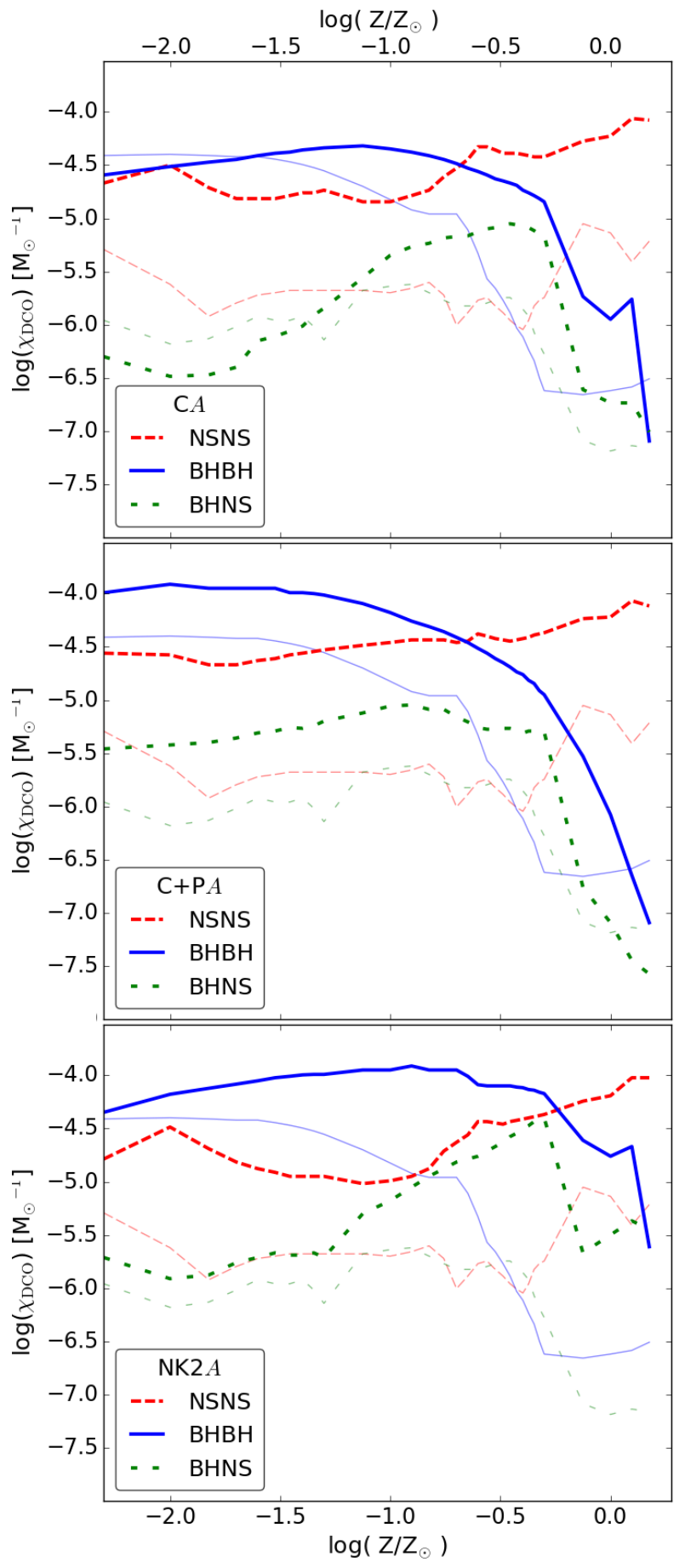

Figure 1. Formation efficiency $\chi_{\mathrm{DCO}}(\mathrm{Z})$ - the number of different types of double compact objects that merge within the Hubble time created per unit of mass formed in stars at certain metallicity $Z$ (in solar units, $Z_{\odot}=0.02$ ) shown for the three cases (labelled $\mathrm{C} A, \mathrm{C}+\mathrm{P} A$ and $\mathrm{NK} 2 A$ as in the original study) identified by $\mathrm{C} 18$ as producing the highest number of local NSNS mergers within the models probed in their study. At the same time, those models were found to overproduce the number of local BHBH mergers. For comparison, $\chi_{\mathrm{DCO}}(\mathrm{Z})$ for their reference model is shown with the thin line in the background. 


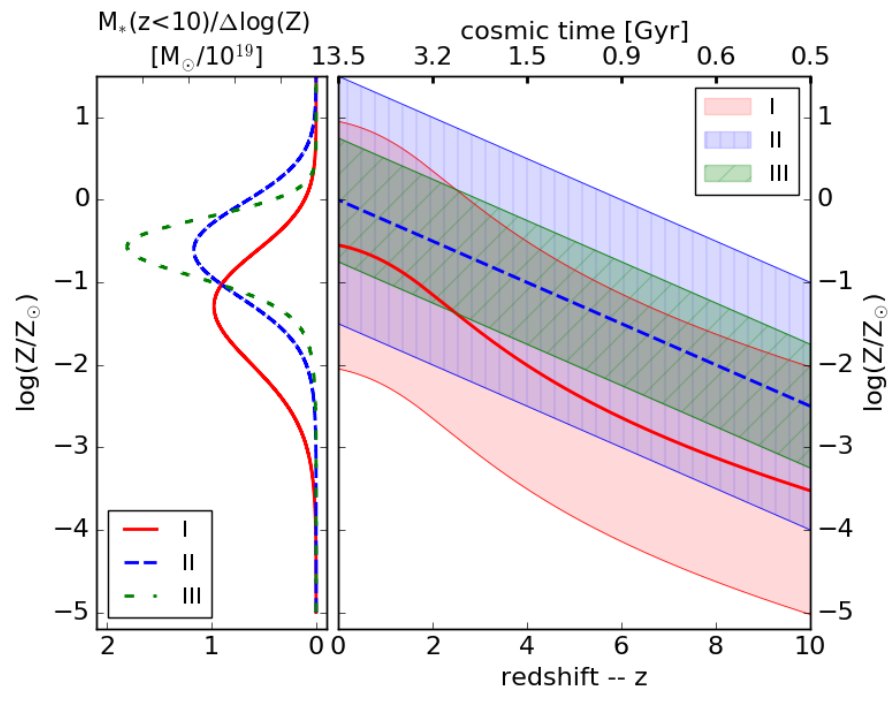

Figure 2. The average metallicity $\mathrm{Z}_{\text {avg }}$ evolution with redshift (thick lines, right panel) for each of the three versions of $\mathrm{f}_{\text {sfr }}(\mathrm{z}, \mathrm{Z})$ distribution describing fraction of star formation that at a given $z$ occurs at a certain metallicity (see sec. 3). Version $I$ is identical to the one introduced in B16 and used by $\mathrm{C} 18 . \mathrm{Z}_{a v g}$ in cases $I I$ and $I I I$ are identical, but the scatter was reduced twice in $I I I$. The shaded regions indicate $3 \sigma$ spread around $\mathrm{Z}_{\text {avg }}$. The left panel shows the distribution of mass formed in stars since redshift $z=10$ at different metallicities for each version of $\mathrm{f}_{\mathrm{sfr}}(\mathrm{z}, \mathrm{Z})$.

efficiency within each metallicity bin centred at one of these values is the same as for that value. We also assume that $\chi(Z)$ of DCOs at $\mathrm{Z}>\mathrm{Z}_{\text {max }}=0.03\left(\mathrm{Z}<\mathrm{Z}_{\min }=0.0001\right)$ are the same as at $\mathrm{Z}_{\max }\left(\mathrm{Z}_{\min }\right)$.

As discussed in 2, the mean metallicity of the star formation used in B16 may lead to an overestimate in the amount of stars forming at low metallicity. This effect would be even stronger if the amount of scatter applied to the assumed $\mathrm{Z}(\mathrm{z})$ relation proves to be too large. Thus, we follow the same procedure as outlined in B16 to calculate $R_{l o c}$, but use three different ways to distribute the cosmic SFR at metallicities, modifying the input $\mathrm{Z}(\mathrm{z})$ relation and hence changing $\mathrm{f}_{\mathrm{sfr}}(\mathrm{z}, \mathrm{Z})$ :

- $I$ - identical to the one from B16

- $I I$ - with higher mean metallicity $\mathrm{Z}_{\text {avg }}\left(\mathrm{Z}_{\text {avg }} \sim \mathrm{Z}_{\odot}\right.$ at $\mathrm{Z}=0$ in contrast to $\mathrm{Z}_{\text {avg }} \sim 0.3 \mathrm{Z}_{\odot}$ assumed in B16)

- $I I I$ - with $\mathrm{Z}_{\text {avg }}$ as in $I I$ but with twice smaller scatter around the mean

Those variations are summarised in fig 2 . In version $I I$ we use the 'low-end' $Z_{\text {avg }}$ introduced by Dominik et al. (2013) who used the MZR found by Erb et al. (2006) and combined it with the average metallicity relation from Pei et al. (1999) to describe its evolution with redshift. We do not argue that the adopted relation provides the best description of the true metallicity evolution of the Universe, but rather use it for its simple form which is sufficient for the purpose of this study. The question of distributing the cosmic SFR at different metallicities clearly deserves a more careful investigation.

\section{RESULTS}

$R_{l o c}$ calculated for the models considered in this study using different $\mathrm{f}_{\mathrm{sfr}}(\mathrm{z}, \mathrm{Z})$ are shown in figure 3 . We also show the reference model (model ref $B$ from $\mathrm{C} 18$ ) for comparison. It can be seen that the applied changes in $\mathrm{f}_{\mathrm{sfr}}(\mathrm{z}, \mathrm{Z})$ generally decrease the $R_{l o c}$ for BHBH and BHNS binaries, while slightly increasing them for NSNS. This is a consequence of both the shape of $\chi_{\mathrm{DCO}}(\mathrm{Z})$ and delay time distribution sharply decreasing for long merger times. Shifting $\mathrm{Z}_{\text {avg }}(\mathrm{z})$ to higher values results in smaller population of low-Z binaries that contribute to the DCO population that merges locally. Decreasing the width of the metallicity distribution strengthens this effect. The NSNS rates in variations $I I$ and $I I I$ increase by a factor of $1.4-2$ with respect to those calculated in $I$. For BHNS binaries this change (decrease) is within a factor of $\sim 3$ (ref model), with hardly any difference between the versions in model $N K 2 A$. The BHBH rates decrease by a factor of $\sim 2($ model $N K 2 A$ ) up to $\sim 12$ ( $r e f$ model in version $I I I)$. Note that these differences are dependent on the $\chi_{\mathrm{DCO}}(\mathrm{Z})$ that results from population synthesis calculations and the examples presented in this study sample only a small fraction of the parameter space involved in such calculations.

In variation $I I I$ for models $C A$ and $C+P A$ the rates for all DCO types are consistent with gravitational waves limits ${ }^{7}$. This is also true for model $C A$ in variation $I I$, where only the mean $\mathrm{Z}_{\text {avg }}$ was increased. For the model $N K 2 A$ the formation efficiency of merging $\mathrm{BHBH}$ remains high up to solar-like metallicities. In this model NSNS and BHBH $R_{l o c}$ observational limits likely cannot be met simultaneously for the same set of evolution-related assumptions for any reasonable model of the SFR history and chemical evolution of the Universe.

In general, mergers of more massive binaries can be detected from larger distances and hence $R_{l o c}$ does not translate directly to the observed frequency of mergers (detection rate), which scales with a combination of masses of merging objects. This effect is mostly important for $\mathrm{BHBH}$ binaries that can form with a wide range of masses. Since the most massive black holes are expected to form at low metallicities, modifications in $\mathrm{f}_{\text {sfr }}(\mathrm{z}, \mathrm{Z})$ have important consequences for the mass (either total or chirp mass $\mathcal{M}_{\text {chirp }}$ ) distribution of merging $\mathrm{BHBH}$ binaries. This distribution extends to higher masses if more (recent) SFR happens at low metallicities and hence the average mass in variations $I I$ and $I I I$ is lower than in I. As a consequence, the decrease in the detection rates for $\mathrm{BHBH}$ estimated for $I I$ or $I I I$ with respect to $I$ would be bigger than in their $R_{l o c}$.

The $\mathcal{M}_{\text {chirp }}$ distribution of merging binaries can be probed with gravitational wave observations which provides additional constraint on our models. Thus, we need to verify if the discussed models agree with the $\mathcal{M}_{\text {chirp }}$ distribution of $\mathrm{BHBH}$ mergers detected so far. In figure 4 we show the detection rate-weighted distribution of BHBH $\mathcal{M}_{\text {chirp }}$ for different models and $\mathrm{f}_{\text {sfr }}(\mathrm{z}, \mathrm{Z})$ variations. The approximate detection rates were calculated using eq. 5 from C18 (see sec. III in Abadie et al. 2010) assuming double neutron star detection range of $170 \mathrm{Mpc}$. It can be seen that in version $I I I$ the detection of BHBH merger with $\mathcal{M}_{\text {chirp }} \gtrsim 30 \mathrm{M}_{\odot}$ is unlikely in all of the models considered in this study, while for $I$ and $I I$ the distribution extends up to $\mathcal{M}_{\text {chirp }} \sim 50 \mathrm{M}_{\odot}{ }^{8}$. While those distributions can be probed by the future observations, for now the observed sample is too small to allow for any firm conclusions

7 12-213 $\mathrm{Gpc}^{-3} \mathrm{yr}^{-1}$ for BHBH (Abbott et al. 2017a), $320-4740$ $\mathrm{Gpc}^{-3} \mathrm{yr}^{-1}$ for NSNS (Abbott et al. 2017c) and an upper limit of 3600 $\mathrm{Gpc}^{-3} \mathrm{yr}^{-1}$ for BHNS/NSBH (Abbott et al. 2016b)

8 the effect of pair instability mass loss was not included in the models presented in this study, however it affects only the most massive BHs $\left(\mathrm{M} \gtrsim 40 \mathrm{M}_{\odot}\right.$ ) forming at very low metallicities and their contribution to the population merging locally is negligible (see Belczynski et al. 2016b) 


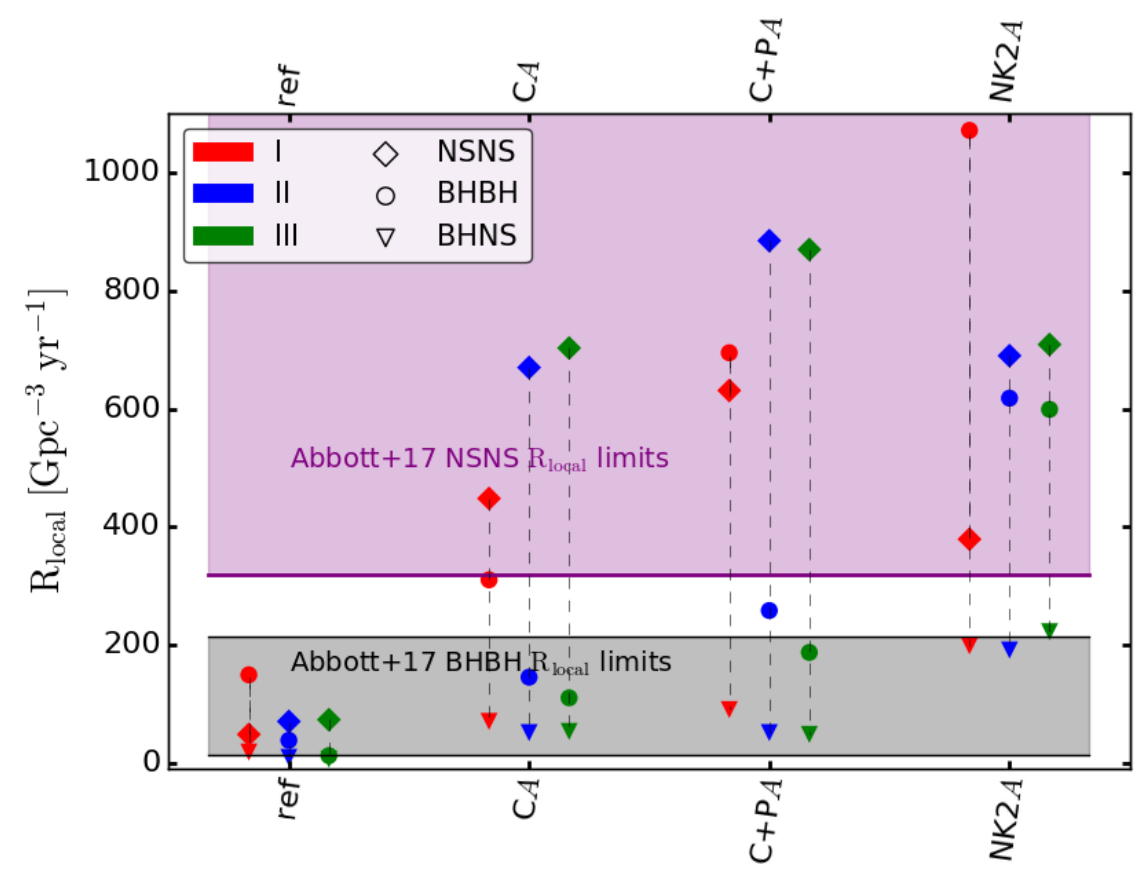

Figure 3. Local merger rate densities $\left(R_{l o c}\right.$ ) of NSNS (trapezium), BHBH (circle), BHNS/NSBH (triangle) calculated for the three models from Chruslinska et al. (2018) that lead to NSNS $R_{l o c}$ above the lower limit implied by the gravitational wave observations (purple line; Abbott et al. 2017c), but the associated BHBH $R_{l o c}$ exceed the corresponding upper limit (black line; Abbott et al. 2017a). We also show their reference model ( $r e f$ ). The colours indicate different assumptions about the distribution of the cosmic star formation rate at different metallicities. The red points (version I) correspond to the model used in the original study, while the blue ones (II) were calculated assuming higher mean metallicity of the star formation. The green set of results was obtained assuming that the scatter around the mean metallicity (described as in II) is twice smaller than in the other two cases.

from the comparison. For instance, two sample KS test performed on each of the model distributions shown in fig. 4 and the observed sample does not allow to rule out any of those distributions at the confidence level higher than $96.4 \%$, with the lowest p-values revealed by models $N K 2 A I(\sim 0.036)$ and $C+P A$ in $I(\sim 0.056)$.

\section{CONCLUSIONS}

Using three models from $\mathrm{C} 18$ as an example, we have demonstrated the importance of the assumptions related to the star formation history and chemical evolution of the Universe for the obtained properties of merging populations of double compact objects, in particular for the estimated merger rate densities. Those models were found to lead to the local NSNS merger rate density consistent with the current limits from gravitational wave observations, at the same time overproducing the number of the local BHBH mergers. One possible solution to this conundrum, as suggested by $\mathrm{C} 18$, is that $\mathrm{BH}$ form with higher natal kicks than assumed in those models, or the common envelope evolution is different for massive $\mathrm{BHBH}$ progenitors than for NSNS progenitors.

Differences in $\mathrm{f}_{\mathrm{sfr}}(\mathrm{z}, \mathrm{Z})$ (or more generally in $\operatorname{SFR}(\mathrm{Z}, \mathrm{z})$ ) induce differences in the properties of the population of merging DCOs observed at a certain redshift. Since the formation efficiency of merging DCOs behaves differently with changing metallicity for different types of systems, the change in $\operatorname{SFR}(\mathrm{Z}, \mathrm{Z})$ affects the ratios of numbers of DCOs of different types and hence their merger rates. The $\mathrm{f}_{\mathrm{sfr}}(\mathrm{z}, \mathrm{Z})$ assumed in the original study likely overestimates the amount of stars forming at low metallicity.

We have shown that when the average metallicity of the star formation is increased to the values more consistent with observations of local galaxies, the number density of local BHBH mergers decreases sufficiently to match the observational limits in one of the models $(C A)$. In section 2 we argued that the amount of scatter around the average used in the original method may be overestimated if metallicity gradients within the regions responsible for the bulk of SFR in the galaxies are sufficiently small. If this scatter is reduced, $\mathrm{R}_{l o c}$ in the model $C+P A$ also meet the gravitational wave limits. In the remaining case the observed $\mathrm{R}_{l o c}$ likely cannot be reproduced simultaneously for all types of DCO by the use of any reasonable $\operatorname{SFR}(Z, z)$ distribution.

Note that the formation efficiency of DCOs is model dependent and so is the change in $\mathrm{R}_{l o c}$ in response to change in $\mathrm{f}_{\text {sfr }}(\mathrm{z}, \mathrm{Z})$. The models used in this study sample only a small part of the parameter space involved in population synthesis calculations. We do not argue that they provide the correct description of the DCO population, but rather use them as a good example showing how the adopted assumptions about $\mathrm{f}_{\mathrm{sfr}}(\mathrm{z}, \mathrm{Z})$ add to degeneracies in the conclusions and final results of those calculations.

Changes in $\operatorname{SFR}(Z, z)$ also have important consequences for the mass (chirp mass) distribution of merging $\mathrm{BHBH}$, which will be sampled with the gravitational wave observations in the future.

Our findings highlight the importance of the choice of a particular way to distribute the cosmic star formation rate across metallicities and time and the need to better understand the uncertainties associated with that choice. Without tighter constraints on this distribution one has to deal with another layer of degeneracy e.g. in the calculated merger rates, besides degeneracies connected to the description of various evolutionary phases of DCO progenitors, which hinders drawing any strong conclusions from studies that aim to use cosmological rates as constraints. 


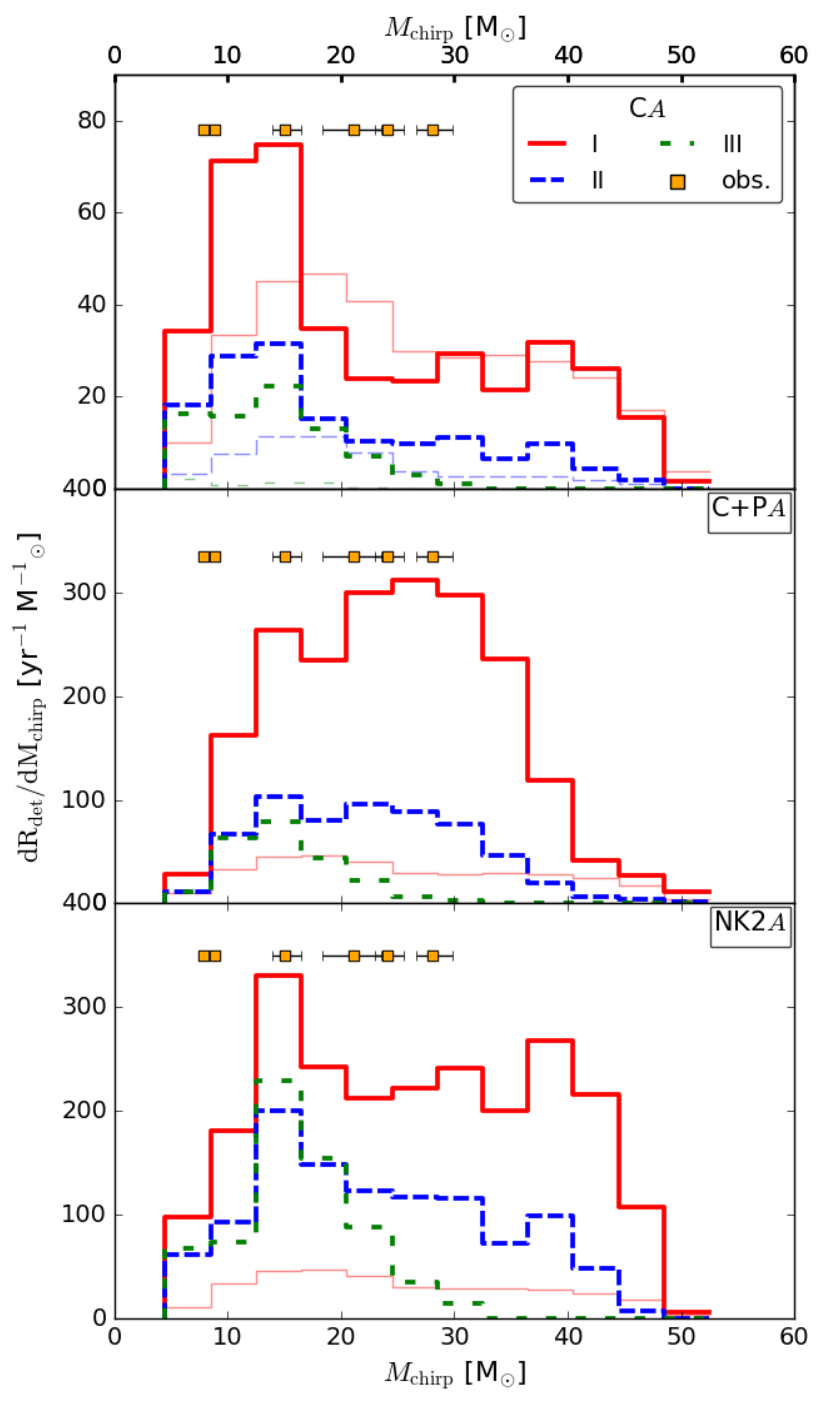

Figure 4. The detection rate $\mathrm{R}_{\text {det }}$ weighted chirp-mass distribution of the $\mathrm{BHBH}$ binaries merging locally for the models considered in this study (different panels) and for the three versions of the distribution of the cosmic star formation rate at different metallicities (different colours). The orange squares mark chirp masses of $\mathrm{BHBH}$ mergers observed in gravitational waves so far (and a candidate detection LVT151012 at $M_{\text {chirp }} \sim 15 \mathrm{M}_{\odot}$; Abbott et al. 2016a, 2017a,b,d). $\mathrm{R}_{\text {det }}$ were calculated assuming detection distances for NS-NS mergers of $170 \mathrm{Mpc}$. The reference model is plotted in the background (thin lines).

\section{ACKNOWLEDGEMENTS}

MC and GN acknowledge support from the Netherlands Organisation for Scientific Research (NWO). KB acknowledges support from the Polish National Science Center (NCN) grants Sonata Bis 2 (DEC-2012/07/E/ST9/01360), OPUS (2015/19/B/ST9/01099), Maestro 2015/18/A/ST9/00746 and LOFT/eXTP 2013/10/M/ST9/00729.
Abbott B. P., et al., 2016b, ApJ, 832, L21

Abbott B. P., et al., 2017a, Physical Review Letters, 118, 221101 Abbott B. P., et al., 2017b, Physical Review Letters, 119, 141101

Abbott B. P., et al., 2017c, Physical Review Letters, 119, 161101

Abbott B. P., et al., 2017d, ApJ, 851, L35

Baraffe I., Heger A., Woosley S. E., 2001, ApJ, 550, 890

Barrett J. W., Gaebel S. M., Neijssel C. J., Vigna-Gómez A., Stevenson S., Berry C. P. L., Farr W. M., Mandel I., 2018, MNRAS, 477, 4685

Belczynski K., Kalogera V., Bulik T., 2002, ApJ, 572, 407

Belczynski K., Kalogera V., Rasio F. A., Taam R. E., Zezas A., Bulik T., Maccarone T. J., Ivanova N., 2008, ApJS, 174, 223

Belczynski K., Bulik T., Fryer C. L., Ruiter A., Valsecchi F., Vink J. S., Hurley J. R., 2010a, ApJ, 714, 1217

Belczynski K., Dominik M., Bulik T., O’Shaughnessy R., Fryer C., Holz D. E., 2010b, ApJ, 715, L138

Belczynski K., Holz D. E., Bulik T., O’Shaughnessy R., 2016a, Nature, 534, 512

Belczynski K., et al., 2016b, A\&A, 594, A97

Belfiore F., et al., 2017, MNRAS, 469, 151

Bray J. C., Eldridge J. J., 2016, MNRAS, 461, 3747

Bray J. C., Eldridge J. J., 2018, MNRAS, 480, 5657

Chruslinska M., Belczynski K., Klencki J., Benacquista M., 2018, MNRAS, 474, 2937

Dominik M., Belczynski K., Fryer C., Holz D. E., Berti E., Bulik T., Mandel I., O'Shaughnessy R., 2012, ApJ, 759, 52

Dominik M., Belczynski K., Fryer C., Holz D. E., Berti E., Bulik T., Mandel I., O'Shaughnessy R., 2013, ApJ, 779, 72

Eldridge J. J., Stanway E. R., 2016, MNRAS, 462, 3302

Eldridge J. J., Stanway E. R., Tang P. N., 2018, MNRAS,

Erb D. K., Shapley A. E., Pettini M., Steidel C. C., Reddy N. A., Adelberger K. L., 2006, ApJ, 644, 813

Giacobbo N., Mapelli M., Spera M., 2018, MNRAS, 474, 2959

Hobbs G., Lorimer D. R., Lyne A. G., Kramer M., 2005, MNRAS, 360, 974

Hurley J. R., Pols O. R., Tout C. A., 2000, MNRAS, 315, 543

Kewley L. J., Ellison S. L., 2008, ApJ, 681, 1183

Klencki J., Moe M., Gladysz W., Chruslinska M., Holz D. E., Belczynski K., 2018, preprint, (arXiv: 1808.07889)

Madau P., Dickinson M., 2014, ARA\&A, 52, 415

Maeder A., 1992, A\&A, 264, 105

Mapelli M., Giacobbo N., Ripamonti E., Spera M., 2017, MNRAS, 472, 2422

Pei Y. C., Fall S. M., Hauser M. G., 1999, ApJ, 522, 604

Peters P. C., 1964, Physical Review, 136, 1224

Poetrodjojo H., et al., 2018, MNRAS, 479, 5235

Sánchez-Menguiano L., et al., 2016, A\&A, 587, A70

Schneider R., Graziani L., Marassi S., Spera M., Mapelli M., Alparone M., Bennassuti M. d., 2017, MNRAS, 471, L105

Stevenson S., Vigna-Gómez A., Mandel I., Barrett J. W., Neijssel C. J., Perkins D., de Mink S. E., 2017, Nature Communications, 8, 14906

Tremonti C. A., et al., 2004, ApJ, 613, 898

Vink J. S., de Koter A., Lamers H. J. G. L. M., 2001, A\&A, 369, 574

de Mink S. E., Belczynski K., 2015, ApJ, 814, 58

This paper has been typeset from a $\mathrm{T}_{\mathrm{E}} \mathrm{X} / \mathrm{L} \mathrm{AT} \mathrm{E}$ file prepared by the author.

\section{REFERENCES}

Abadie J., et al., 2010, Classical and Quantum Gravity, 27, 173001

Abbott B. P., et al., 2016a, Physical Review X, 6, 041015 